\title{
Das St. Galler Projekt: Vom Projekt zum Modell?
}

\author{
Verwaltungsrat und Geschäftsleitung des Kantonsspitals St. Gallen haben beschlos- \\ sen, die Hausarztmedizin im Zentrumsspital zu implementieren. Dafür wurde die \\ Stelle eines Leitenden Arztes für Hausarztmedizin geschaffen und hausarztrelevante \\ Ausbildungsbereiche wurden in bestehende Strukturen integriert.
}

\author{
Christian Häuptlea, \\ Christa Meyenberger ${ }^{b}$, \\ Peter Greminger \\ a Dr. med., Facharzt für \\ Allgemeine Medizin FMH; \\ Leitender Arzt Hausarztmedi- \\ zin, Allgemeine Innere \\ Medizin, Kantonsspital \\ St. Gallen \\ b Prof. Dr. med., Chefärztin \\ Gastroenterologie/ \\ Hepatologie, Kantonsspital \\ St. Gallen \\ c Prof. Dr. med., Chefarzt \\ Allgemeine Innere Medizin, \\ Kantonsspital St. Gallen
}

Korrespondenz:

Dr. med. Christian Häuptle Leitender Arzt Hausarztmedizin Allgemeine Innere Medizin Kantonsspital St.Gallen CH-9007 St.Gallen

christian.haeuptle@kssg.ch

\section{Einleitung}

Die ärztliche Grundversorgung, sichergestellt durch die Hausarztmedizin, ist ein Grundpfeiler und ein Qualitätsmerkmal des schweizerischen Gesundheitswesens. Es ist entscheidend, auch künftig die hausärztliche Grund- und Notfallversorgung in den Kantonen zu garantieren. Verschiedene Faktoren stellen das bis anhin gutfunktionierende System der Grundversorgung vor einige Herausforderungen:

- Die Demographie der Hausärzte* lässt in den nächsten Jahren, vor allem in ländlichen Gebieten, Praxen verwaisen.

- Der generelle Mangel an Ärzten, bedingt durch die begrenzten Ausbildungsplätze an den Universitäten, trifft auch die Grundversorgung.

- Durch die Feminisierung des Berufes wird ein Teil der Ärztinnen in Teilzeitarbeit tätig sein, was den Bedarf an weiteren Ärztinnen und Ärzten erhöht.

- Das Spital ist mit seinen attraktiven Anstellungsbedingungen (geregelte Arbeitszeit, sicherer Arbeitsplatz, befriedigender Lohn, anregendes $\mathrm{Ar}$ beitsumfeld, Arbeit im Team, gute Karrieremöglichkeiten, keine Querelen mit den Krankenkassen usw.) zu einer klaren Alternative gegenüber der freien Praxistätigkeit geworden.

- Die aktuellen Rahmenbedingungen sind für die Hausarztmedizin, trotz wiederholter Beteuerungen der Politik, die Grundversorgung zu stärken, in den letzten Jahren zunehmend schlechter geworden. Es ist nicht erstaunlich, dass bei einer Befragung von Ärzten durch die Fachhochschule St. Gallen (FHSG) im Jahre 2006 64\% der Befragten gerade diese Tatsache als Grund anführten, weshalb sie ihre Zukunft nicht in der Grundversorgung sehen. Die Verbesserung der hausärztlichen Rahmenbedingungen ist aber abhängig vom politischen Willen, diese auch durchzusetzen.

- Die ärztliche Weiterbildung ist stark auf die Universitäts- und Zentrumsspitäler ausgerichtet. In diesem spezialisierten Umfeld wird die Hausarztmedizin nur marginal wahrgenommen und das

\section{Zusammenfassung}

Um die hausärztliche Weiterbildung zu stärken und die hausärztliche Tätigkeit nachhaltig zu positionieren, wurde ein Projekt entwickelt, das diese Anliegen kontinuierlich und effizient umsetzen soll. In der Weiterbildung geschieht dies durch ein attraktives Praxisassistenzangebot und ein individuell zusammenstellbares Curriculum. In einem persönlichen Laufbahngespräch wird mit der Kandidatin oder dem Kandidaten eine massgeschneiderte Weiterbildung evaluiert. Durch den Einbezug der Hausarztmedizin in den Klinikalltag, namentlich durch die klinische Arbeit bei der Visitenbegleitung, kann das Interesse an der Grundversorgung bei den Assistenten* geweckt und generell das Verständnis für die hausärztliche Tätigkeit gefördert werden. Das rege Interesse am Weiterbildungsangebot sowie die zunehmende Kooperation der Spital- und Hausärzte sind Hinweise, dass die Startphase geglückt ist. Es gilt nun, die Strukturen zu festigen, das Angebot weiter auszubauen und die Kontinuität sicherzustellen.

Wissen um die Grundversorgung und deren Inhalte wird dort immer kleiner. So erstaunt es nicht, dass $80 \%$ der Ärzte, welche die hausärztliche Tätigkeit aufnehmen wollen, während ihrer Weiterbildung keine spezifische Ausbildung in Hausarztmedizin durchlaufen haben.

Mit dem Ziel, die hausärztliche Weiterbildung zu verbessern, hat eine Gruppe von Haus -und Spitalärzten 2006 gemeinsam ein Weiterbildungsprojekt entwickelt. Dieses soll die Attraktivität und Qualität der Hausarzt- 
medizin fördern und die Hausarztmedizin innerhalb der spezialisierten Medizin neu positionieren. Das ausgearbeitete Konzept wurde in Form einer Interpellation, die von allen wichtigen Parteien des Kantonsrates des Kantons St. Gallen unterstützt wurde, 2006 der Regierung eingereicht. Im September 2007 genehmigte der Kantonsrat ohne Gegenstimme den regierungsrätlichen Bericht zur Weiterbildung zur Hausarztmedizin im Kanton St. Gallen. Damit war die politische Legitimation dieses Projektes gegeben und die Finanzierung gesichert. Offiziell wurde das Projekt am 1. Januar 2008 gestartet.

\section{Das Projekt}

Das Projekt beinhaltet 2 Schwerpunkte:

- Die hausärztliche Aus- und Weiterbildung

- Die Implementierung der Hausarztmedizin am Zentrumsspital

Das Projekt wurde bewusst am Zentrumsspital St. Gallen platziert. Man wollte die vielfältigen Ressourcen und Synergien des Zentrums nutzen. Zudem sollte die Hausarztmedizin dort platziert sein, wo die ärztliche Weiterbildung intensiv vermittelt wird und wo auch ein vielfältiger Kontakt mit den «Kunden», also den Assistenten, gepflegt werden kann. Die Regionalspitäler St. Gallens sind in dieses Projekt eingebunden.

\section{Die Aus- und Weiterbildung}

Hier sind 4 Bereiche definiert, die bisher unter dem Begriff «Kleeblatt» zusammengefasst wurden:

- Praktika für Studentinnen und Studenten in Hausarztpraxen;

- Praxisassistenz mit kantonaler Finanzierung;

- Curriculum (Weiterbildungsgang) für angehende Hausärzte;

- Supervision und Wegbegleitung der Weiterbildung.

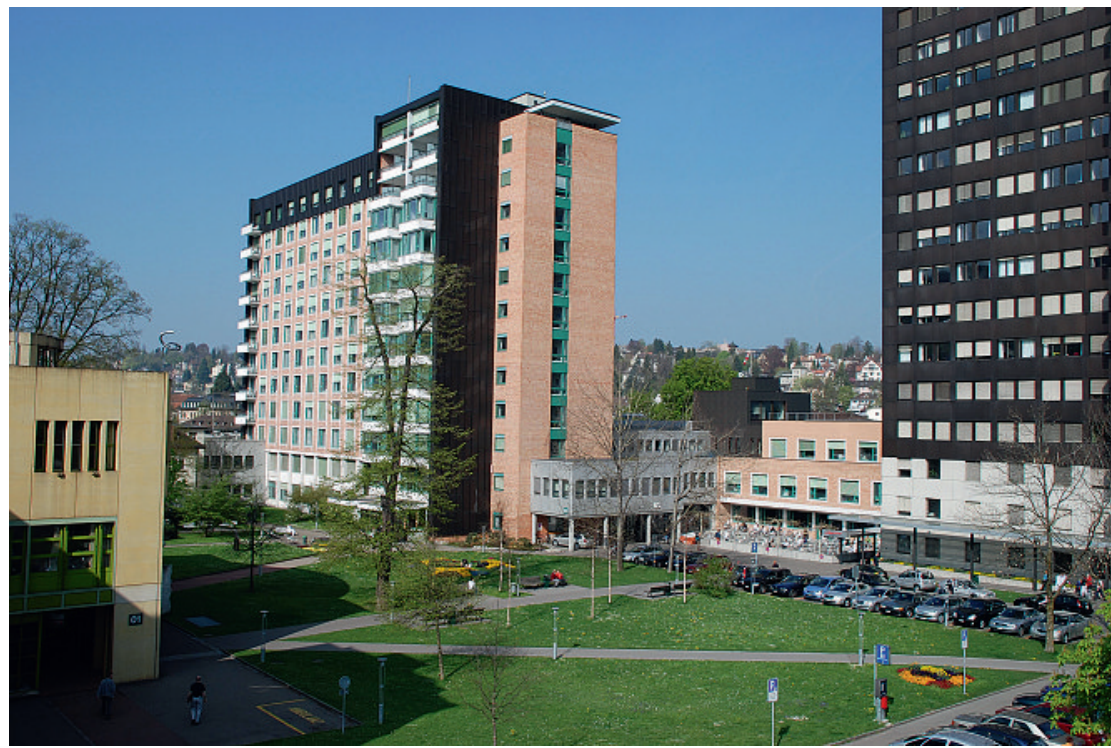

Das Kantonsspital St. Gallen hat die Hausarztmedizin in den Klinikalltag integriert.

\section{Ausbildung/Praktika}

Schon lange werden Studierende der Medizin in sanktgallischen Hausarztpraxen und in Kursen am Kantonsspital St. Gallen ausgebildet. Ziel ist es, die Auszubildenden mit der Hausarztmedizin besser vertraut zu machen und sie für eine Weiterbildung in Hausarztmedizin zu motivieren.

\section{Praxisassistenz}

Nach der kantonalen Vorgabe können jedes Jahr vier 6-monatige Praxisassistenzen vergeben werden. Die 6-monatige Praxisassistenz zu 100\% Arbeitszeit kann auch in einer 50\%-igen Teilarbeitszeit für $1 \mathrm{Jahr}$ absolviert werden. Damit sollen auch Ärzte mit Kindern die Möglichkeit haben, an dieser Weiterbildung teilzunehmen. Die Praxisassistenz ist eine Weiterbildungsstelle und wird nach den Richtlinien der Praxisassistenz des KHM (Kollegium für Hausarztmedizin) durchgeführt. Grundsätzlich soll die Praxisassistenz nach einer vorgängig 3-jährigen klinischen Weiterbildung angetreten werden. Bei einer zweijährigen klinischen Vorbildung kann die in der Praxisassistenz vorgesehene Praxisvertretung nur unter der Supervision eines Lehrarztes geleistet werden, da die kantonale Gesetzgebung eine 3-jährige Weiterbildung für eine Praxisvertretung verlangt. Die Weiterbildung wird durch Lehrärzte, die in den Kursen des KHM ausgebildet wurden, vermittelt.

Die Praxisassistenz wird vorwiegend durch den kantonalen Beitrag finanziert. Die Praxisassistenten erhalten den vollen Lohn nach den kantonalen Richtlinien.

Der Lehrpraktiker bezahlt 2000 Franken im Monat. Der Kanton St. Gallen budgetiert für die vier 6-monatigen Praxisassistenzstellen 233000 Franken. Die Lehrärzte beteiligen sich mit maximal 48000 Franken an dieser Weiterbildung.

Die Anstellungsbedingungen wurden möglichst einfach gestaltet. Die Praxisassistenten (wie auch die Curriculum-Assistenten) werden, falls sie nicht schon als Assistenten am Kantonsspital St. Gallen arbeiten, als reguläre Assistenten des Kantonsspitals angestellt. Sie erhalten so den Versicherungsschutz und die üblichen Sozialleistungen wie ihre anderen Kollegen am Spital. Die Praxisassistenten sind dem Fachbereich Allgemeine Innere Medizin (AIM) angegliedert, werden durch das Gesundheitsdepartement fremdfinanziert und figurieren ausserhalb der regulären Assistenzstellen des Fachbereichs AIM. Die Praxisassistenten werden - wie bei einer Praxisvertretung - dem Lehrpraktiker «ausgeliehen». Es kommt somit zu einem Vertrag zwischen Lehrarzt und Spital. Im Vertragsanhang werden die Aufgaben der Praxisassistenten geregelt. Dieses einfache Anstellungsverfahren benötigt keine Zusatzverträge.

Die Praxisassistenz unterliegt keiner Rückerstattungspflicht. Es kann ja nicht angehen, dass man jungen Ärzten die Möglichkeit gibt, Einblick in die ärztliche Grundversorgung zu erhalten, um sie für dieses 
Berufsziel zu gewinnen, sie aber bei einem allfälligen negativen Entscheid dafür finanziell bestraft.

\section{Curriculum}

Das Curriculum soll zukünftigen Hausärzten die Möglichkeit geben, sich jene Fähigkeiten aus einem Fachgebiet anzueignen, die sie in ihrer Tätigkeit kompetent und eigenverantwortlich einsetzen können. Diese Curriculumsstellen können in einem Fachgebiet der persönlichen Wahl angetreten werden. Im Unterschied zu diese Assistenten vornehmlich im Ambulatorium sowie im Notfalldienst einzusetzen Diese Forderung der hausarztspezifischen Weiterbildung wird dadurch erleichtert, weil die Curriculumsstelle den klinikeigenen Assistentenpool nicht belastet und ja grösstenteils fremdfinanziert wird.

Die Finanzierung geschieht analog der Praxisassistenz: die Assistenten erhalten ihren üblichen Lohn, die weiterzubildende Stelle bezahlt pro Monat 2000 Franken und die restlichen Kosten übernimmt der Kanton.

\section{Die Assistenten haben im Spital einen Ansprechpartner in Hausarztmedi- zin, sie kennen ihn von der Visite und verschiedenen Lehrveranstaltungen}

den regulären Facharztstellen, die oft nur angehenden Fachärzten vorbehalten sind und eine längere Assistenz erfordern, sind die Curriculumsstellen auf 6 Monate zu $100 \%$ oder 12 Monate zu $50 \%$ je Fachbereich limitiert. Die Curricula werden zu drei mal sechs Monaten, insgesamt für 18 Monate, angeboten. Oft wird das Curriculum mit einer 6-monatigen Praxisassistenz ergänzt, so dass ein gesamter Weiterbildungsgang zwei Jahre dauert. Damit ist eine vollständige Ausbildung nach 3-jähriger klinischer Vorbildung und 2-jähriger spezieller hausärztlicher Weiterbildung in fünf Jahren möglich. Das Curriculum kann auch in 6-monatigen Weiterbildungsmodulen absolviert werden. Es müssen nicht zwingend 18 Monate belegt werden, um an diesem Weiterbildungsgang teilnehmen zu können. Grund dafür ist die Tatsache, dass sich gut die Hälfte der Assistenten erst während ihrer klinischen Weiterbildung dazu entschliesst, in die Hausarztmedizin zu gehen, und daher nicht das gesamte Curriculum benötigt (FHS 2006). Junge Ärzte, die sich für das gesamte Curriculum entscheiden, erhalten den Vorzug. Mit dieser Lösung kann die Weiterbildung individuell evaluiert werden. Das Curriculum kann in einem Fachbereich des Zentrumsspitals, in einer klinischen Abteilung der Regionalspitäler des Kantons sowie auch grundsätzlich in spezialisierten Arztpraxen absolviert werden.

Die Einrichtung und Evaluation des Curriculums ist aufwendig. Im persönlichen Gespräch mit den Chefärzten der verschiedenen Fachrichtungen wird geklärt, ob sie bereit sind, eine solche Weiterbildungsstelle in ihrem Fachgebiet einzurichten. Bei Zusage wird ein verantwortlicher Lehrer für die Betreuung der Weiterzubildenden, meist ein Kaderarzt, bestimmt. Die Lernziele des Curriculums werden umschrieben und die Lerninhalte definiert. Da in 6 Monaten Curriculumszeit sich die Weiterzubildenden vor allem hausarztspezifische Fähigkeiten in diesem speziellen Fachgebiet aneignen sollen, ist es zentral, dass diese Lerninhalte auch wirklich vermittelt werden. So sind
Beim Curriculum besteht im Gegensatz zur Praxisassistenz eine Rückerstattungspflicht. Dies bedeutet, dass pro rata temporis der Weiterbildung im Curriculum sich der Arzt verpflichtet, bis maximal 5 Jahre, spätestens aber nach 7 Jahren der abgeschlossenen Weiterbildung im Kanton in der Grundversorgung zu praktizieren. Ein spezielles Reglement legt im einzelnen das Vorgehen sowie die verschiedenen Ausnahmen dar.

Die Praxisassistenz wie auch das Curriculum werden regelmässig evaluiert.

\section{Supervision und Wegbegleitung}

Die regierungsrätliche Verordnung zur Hausarztmedizin sieht vor, dass die Praxisassistenz und das Curriculum von einem Tutorenteam begleitet werden. Dieses hat die Aufgabe, geeignete Kandidaten zu rekrutieren, die Vereinbarungen sowie die Zielsetzungen mit den angehenden Grundversorgern und den Weiterbildungspartnern zu prüfen (Qualitätskontrolle) und ein Tutorat sowie Supervisionsangebot für die Weiterzubildenden anzubieten. Das Tutorenteam setzt sich aus einem Hausarzt oder einer Hausärztin (Vorsitz), einem Chefarzt des Zentrumsspitals sowie einem Chefarzt eines sanktgallischen Regionalspitals zusammen. Das Tutorenteam berichtet periodisch dem Gesundheitsdepartement über seine Tätigkeit. Im regierungsrätlichen Bericht wird festgehalten, dass geeignete Kandidatinnen und Kandidaten für die Praxisassistenz und das Curriculum evaluiert werden sollen. Dies geschieht im persönlichen Gespräch, bei dem der optimale, massgeschneiderte Weiterbildungslehrgang entwickelt wird. Diese Laufbahnberatung soll möglichst jene Faktoren mitberücksichtigen, die eine Evaluation beeinflussen, wie z.B. die persönlichen Lebensumstände, Neigungen, Lebensentwürfe usw.

Das Tutorenteam wird pauschal für seine Arbeit mit 50000 Franken jährlich entschädigt.

Bei Vollauslastung des gesamten Projekts mit Praxisassistenz, Curriculum sowie den Kosten des Tuto- 
renteams ergibt sich ein finanzieller Beitrag des Kantons von 975000 Franken. Zusätzlich kommen die Einnahmen von den Lehrarztpraxen sowie der anderen Weiterbildungsstätten von jährlich 240000 Franken hinzu. Total belaufen sich die Kosten maximal auf 1215000 Franken.

\section{Hausarztmedizin am Kantonsspital St. Gallen}

Neben der Aufgabe der Aus- und Weiterbildung wurde vom Verwaltungsrat und der Geschäftsleitung des Kantonsspitals St. Gallen beschlossen, es sei die Hausarztmedizin im Zentrumsspital zu implementieren. Dafür wurde die Stelle eines Leitenden Arztes für Hausarztmedizin geschaffen. Diese Stelle umfasst heute ein 50\%-iges Arbeitspensum. Der Leitende Arzt für Hausarztmedizin ist für die Umsetzung der Aus- und Weiterbildung in Hausarztmedizin verantwortlich und mit dem Chefarzt der Allgemeinen Inneren Medizin für die Implementierung der Hausarztmedizin am Spital zuständig. So wurden Tätigkeitsbereiche im Spital untersucht, die für die Zusammenarbeit zwischen Spital- und Hausarzt zentral sind. Die hausarztrelevante Aus- und Weiterbildung am Spital wurde in den bestehenden Strukturen eingerichtet z. B. im praktischen Seminar für Assistentinnen und Assistenten der Inneren Medizin. Neu wurde die Visitenbegleitung des Leitenden Arztes für Hausarztmedizin eingeführt. Die Beratung des Spitals in Fragen der Hausarztmedizin sowie die Förderung der Kommunikation und Koordi-

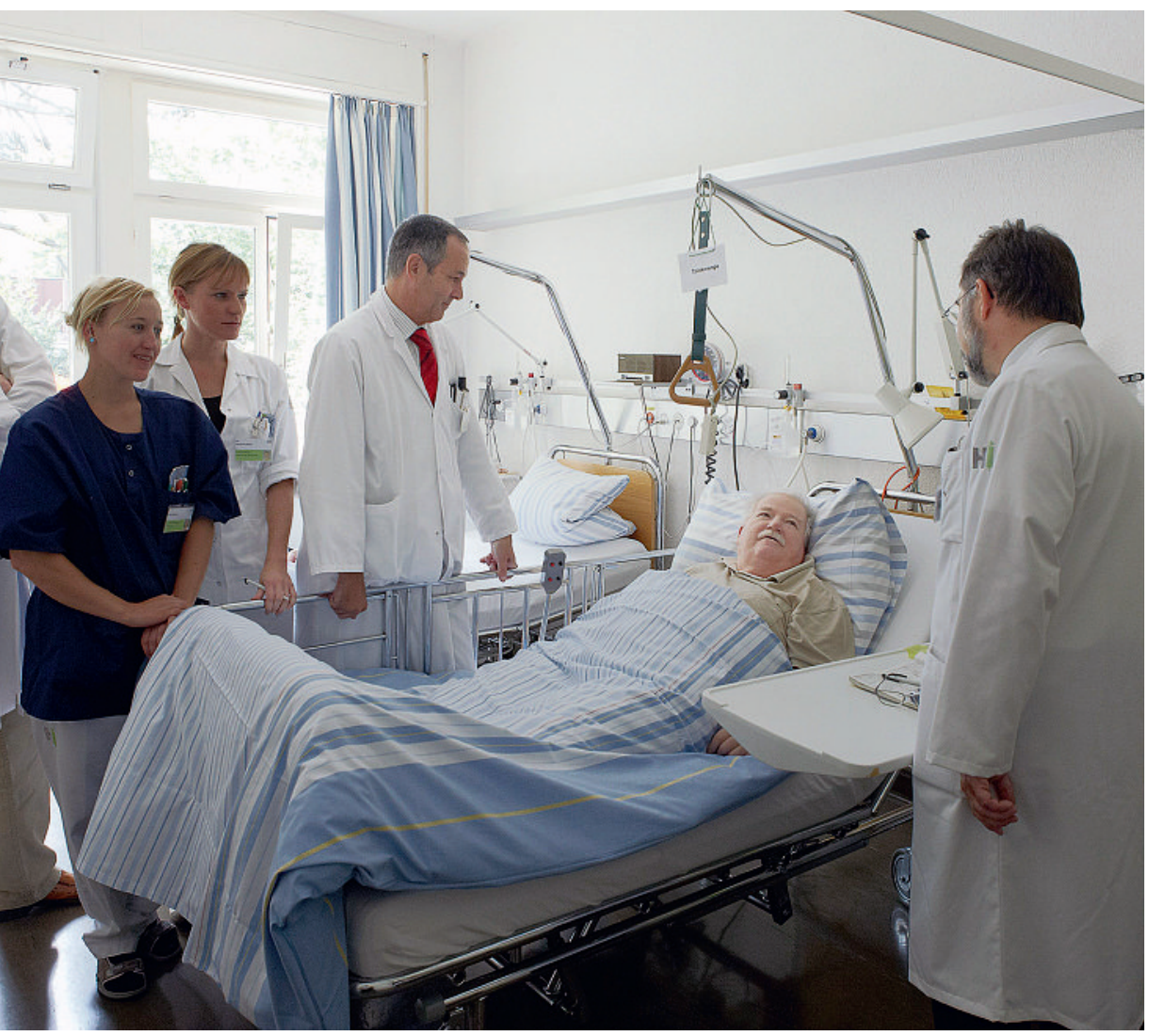

Die Visitenbegleitung des Leitenden Arztes für Hausarztmedizin ist ein wichtiger Bestandteil der hausarztrelevanten Aus- und Weiterbildung. nation zwischen Spital und Hausarztpraxis sind weitere Aufgaben.

Durch diese Präsenz konnte erreicht werden, dass die Hausarztmedizin im Klinikalltag überhaupt wahrgenommen wird. Durch persönliche Kontakte mit Chef- und Kaderärzten können Fragen der Kommunikation und Koordination zwischen Spital und Grundversorgung effizient und einfach gelöst werden. Die Assistenten haben im Spital einen Ansprechpartner in Hausarztmedizin, sie kennen ihn von der Visite und von den verschiedenen Lehrveranstaltungen. Diese hausärztliche Präsenz im Klinikalltag ist ein entscheidender Faktor für das Gelingen des Projekts.

\section{Erste Erfahrungen}

Die Verknüpfung der beiden Projektschwerpunkte, die Weiterbildung mittels Praxisassistenz und Curriculum einerseits und die Implementierung der Hausarztmedizin am Zentrumsspital andererseits, erweist sich als fruchtbar und erschliesst Synergien. Die Präsenz des Leitenden Arztes gibt der Hausarztmedizin im Spital ein «Gesicht». Vor allem die Visitenbegleitung, meistens auf der Chefarztvisite der Allgemeinen Inneren Medizin, ist eine hervorragende Möglichkeit, in direkten Kontakt mit den Assistenten wie auch mit den Kaderärzten zu kommen. Da geht es um «das Kerngeschäft» jeder ärztlichen Tätigkeit: um die Krankheit und das Leiden der Patienten. Hier kann man unmittelbar die hausärztliche Sichtweise anbringen, am Teaching teilnehmen und dabei selbst viel lernen. In gegenseitigem Respekt hat sich so ein «Netzwerk» zwischen Kaderarzt, Assistenzarzt und Hausarzt entwickelt Die Kontaktnahme mit den Weiterzubildenden am Krankenbett führt zu einem Interesse an der Grundversorgung, was die Rekrutierung sehr erleichtert. Die Mitarbeit bei klinikinternen Weiterbildungsprogrammen sowie das regelmässige Vorstellen des Projekts, auch in den Regionalspitälern, unterstützt das Bemühen, geeignete Kandidatinnen und Kandidaten anzusprechen.

Die persönliche Laufbahnberatung ist ein zentrales Instrument für die Umsetzung des Projektes. Diese Beratung wird nicht nur von Interessenten für das Projekt selber beansprucht, sondern auch von Kolleginnen und Kollegen, die sich für eine selbständige ambulante Tätigkeit in einem Fachgebiet interessieren. Waren es im Jahr 2008 ca. 25 bis 30, so waren es 2009 schon über 30 Beratungen, die sich öfters über mehrere Gesprächsrunden hinzogen.

In den ersten 2 Jahren des Projektes konnten 13 Praxisassistenzen durchgeführt werden. Die grössere Anzahl Praxisassistenz war möglich, weil die Curriculumsstellen noch nicht evaluiert waren und man vermehrte Praxisassistenzstellen anbieten konnte. Das Curriculum, welches einen deutlich grösseren konzeptionellen Aufwand erfordert, ist ebenfalls auf gutem Wege. Drei Curriculumsstellen konnten etabliert werden: eine Stelle in der ORL, eine Dermatologiestelle sowie eine Ophthalmologiestelle. Geplant ist für 
das Jahr 2010 eine weitere Weiterbildungsstelle in der Gastroenterologie mit Schwerpunkt Sonographie. In diesem Lehrgang sollen die angehenden Hausärzte die Gelegenheit erhalten, ihre Ultraschall-Kenntnisse in Abdominalsonographie zu komplettieren und den Fachausweis für Abdominalsonographie zu erhalten. Weitere Curriculumsstellen werden je nach Nachfrage timation, der zuverlässige Rückhalt im Gesundheitsdepartement, die Aufnahme der Hausarztmedizin am Zentrumsspital durch den Verwaltungsrat und der Geschäftsleitung sowie die kollegiale und partnerschaftliche Aufnahme durch die Chefärztekonferenz des Kantonsspital St.Gallen sind unverzichtbare Elemente, die ein erfolgreiches Umsetzen erst möglich machen.

\section{Weiterbildung durch Praxisassistenz und Curriculum sowie Implementierung der Hausarztmedizin am Zentrumsspital erweisen sich als fruchtbar und erschliessen Synergien}

entwickelt und angeboten. Sicherlich wird ein Curriculum in Chirurgie/Orthopädie entwickelt, da in der neuen Weiterbildungsverordnung für die Allgemeine Innere Medizin eine obligatorische Weiterbildung in einem chirurgischen Fach ja leider völlig fehlt und Kenntnisse in der Chirurgie/Orthopädie in der Grundversorgung zwingend sind. Hier soll in der Orthopädie wie auch in der Chirurgie ein 6-monatiges Curriculum angeboten werden.

Zwei Personen stehen in der Curriculumsausbildung. Drei Curriculumsstellen sind für das Jahr 2010 schon belegt. Die Praxisassistenzstellen sind für 7 Kandidatinnen und Kandidaten definitiv vergeben.

\section{Fazit}

Die Startphase des St.Galler-Projekts scheint geglückt zu sein. Ist es auf dem Weg vom Projekt zum Modell? Es ist noch zu früh, um dies beantworten. Es wird aber weiterhin wichtig sein, auch in Zukunft besonnen und Schritt für Schritt die Weiterbildung auszubauen und diese nachhaltig zu festigen. Die politische Legi-
Und schliesslich sind es enthusiastische Kolleginnen und Kollegen aus Spitalmedizin und Grundversorgung, die in exemplarischer Weise aufgezeigt haben, dass eine konstruktive Zusammenarbeit zwischen Spitzenmedizin und Grundversorgung immer noch möglich ist.

\section{Dank}

Ein solches Projekt ist Teamarbeit. Der Dank geht an die Kollegen aus der Praxis und der Kontaktgruppe, namentlich an Herrn Dr. Christian Schibler und Herrn Dr. Andreas Hartmann, für ihre grosse Mitarbeit, an Frau Regierungsrätin Heidi Hanselmann für ihre politische und Herrn Dr. Peter Wiedersheim für seine standespolitische Unterstützung.

Ein spezieller Dank gebührt Herrn lic. rer. publ. Hans Leuenberger, Direktor und CEO des Kantonsspitals St.Gallen. Er hat mit grosser Begeisterung und kompromisslosem Einsatz diesem Projekt zur Realisierung verholfen, indem er «sein Haus» für die Hausarztmedizin öffnete. 